\title{
Ocular involvement in melioidosis: a 23-year retrospective review
}

\author{
Sasi Yaisawang ${ }^{1 *}$ D, Somkiat Asawaphureekorn', Ploenchan Chetchotisakd ${ }^{2,3}$, Surasakdi Wongratanacheewin ${ }^{3,4}$ \\ and Peerapat Pakdee
}

\begin{abstract}
Background: Ocular involvement in melioidosis is rare and has devastating outcomes. Although there have been few reports on the condition, Khon Kaen, a city in northeast Thailand, has been called the "capital of melioidosis" due to the high prevalence of the condition in the region. We retrospectively reviewed all admitted cases of melioidosis with ocular involvement from the two largest hospitals in Khon Kaen. We reviewed cases from Srinagarind Hospital (a university hospital) of patients admitted between 1993 and 2016 and from Khon Kaen Hospital (a provincial hospital) of patients who presented from 2012 to 2016.
\end{abstract}

Results: We identified 16 cases of ocular involvement. Eight of these cases were proven from positive culture, and the remaining eight were implied from high melioidosis titer. The prevalence was estimated as being from 0.49 to $1.02 \%$. Most patients had underlying diseases $(14,88 \%)$, of which diabetes mellitus was the most prevalent $(12,75 \%)$. Nine cases $(56 \%)$ were part of disseminated septicemia. Patients suffered from blindness in 11 (73\%) of the 15 cases in which visual acuity was recorded. Orbital cellulitis was the most common manifestation $(7,44 \%)$ followed by endophthalmitis $(4,25 \%)$. Interestingly, all patients with necrotizing fasciitis (100\%) developed septic shock as a consequence. In most of the cases, patients underwent surgery $(13,81 \%)$ including incision and drainage, debridement, and pars plana vitrectomy. Despite appropriate management, the visual outcomes were disappointing $(9,64 \%)$.

Conclusion: To summarize, ocular melioidosis is a highly destructive disease. Early detection and prompt surgical management may reduce morbidity and mortality from septic shock.

Keywords: Melioidosis, Burkholderia pseudomallei, Glanders, Orbital cellulitis, Endophthalmitis

\section{Background}

Melioidosis is caused by a gram-negative, motile, non-spore forming facultative anaerobic bacillus known as Burkholderia pseudomallei. The organism is found in soil and surface water and is widely distributed in Southeast Asia, especially in northeast Thailand and northern Australia [1].

Melioidosis presents with broad spectrums of clinical presentations and organ involvement. However, there are few case reports of ocular involvement in melioidosis, and most of these are single-case report or small case series.

\footnotetext{
*Correspondence: whatsfor@gmail.com; sasi.y@kkumail.com

${ }^{1}$ Department of Ophthalmology, Faculty of Medicine, Khon Kaen University, Khon Kaen, Thailand

Full list of author information is available at the end of the article
}

In northeast Thailand, there are around 2000 culturepositive melioidosis cases per year [2]. Khon Kaen, one of the largest cities in northeast Thailand, has been called "the capital of melioidosis" due to the high prevalence of the disease in the region. Ocular involvement in these cases has not been investigated. The primary objective of this study was to estimate the prevalence and investigate ocular manifestations of melioidosis in Khon Kaen. Management and visual outcomes in these patients were also reviewed.

\section{Results}

We identified 16 cases of ocular involvement, 13 out of the 1270 melioidosis cases admitted to Srinagarind Hospital (prevalence 1.02\%; 95\% confidence interval from 
0.58 to $1.76 \%$ ) and three out of the 607 admitted cases at Khon Kaen Hospital (prevalence 0.49\%; 95\% confidence interval from 0.10 to $1.51 \%)$. Overall, the estimated prevalence of ocular involvement in cases of melioidosis was from 0.49 to $1.02 \%$ (Table 1 ).

Of those 16 cases, there were 8 with positive cultures. In the remaining eight cases, melioidosis was implied from the high titer for melioidosis in the bloodstream. Clinical descriptions of all cases are summarized in Table 2.

Baseline characteristics of the patients were comparable to general melioidosis patients. The male to female ratio was 3 to 1 with a median age of 50.5 years old (3970 ). The most common occupation was farmer (nine cases, 56\%). Most patients had underlying diseases (14 cases, $88 \%$ ), of which diabetes mellitus was the most common (12 cases, 75\%). Ocular involvement was part of dissemination in nine cases $(56 \%)$, which were classified as disseminated septicemic melioidosis.

The majority of ocular melioidosis patients (10 cases, 63\%) presented with eye symptoms. Interestingly, the other six cases initially presented with fever or a headache. Out of the 15 cases for which there were records of visual acuity, 11 (73\%) presented with blindness. The ocular manifestations of melioidosis were classified as orbital cellulitis (seven cases, 44\%), preseptal cellulitis (two cases, 13\%), endophthalmitis (four cases, 25\%), panophthalmitis (two cases, 13\%), and panuveitis (one case, 6\%).

In most cases, the definitive management was surgery (13 cases, 81\%) including incision and drainage, debridement (eight cases, 62\%), pars plana vitrectomy (three cases, 23\%), and enucleation (two, 15\%). There were only three cases $(19 \%)$ in which the patients were able to be treated without surgery.

Despite adequate surgical intervention, the visual outcomes of ocular melioidosis were disappointing. Out of the 14 cases for which there were records of final visual acuity, nine (64\%) patients ended up legally blind. Three of these patients (20\%) presented with no light perception at the beginning, two had to be enucleated, two (14\%) were stable, and two (14\%) had progressive loss of vision. Patients had improved vision after treatment in only five cases (36\%).

\section{Discussion}

To our knowledge, this is the first and largest case series of ocular involvement in melioidosis. A comprehensive literature review revealed only 14 cases from 12 reports [3-14], including 7 cases of orbital cellulitis (50\%), 3 cases of endophthalmitis (21\%), 3 cases of corneal ulcer (21\%), and 1 case of acute dacryocystitis (7\%). Most of the reports were single-case reports, and the largest one had only three cases.

In Thailand, especially in the northeast, there has been an increase in the reported cases of melioidosis. This is likely due to increasing awareness of the condition and increased sensitivity of the technology used to detect the organism. The mortality rate in these areas is around $40 \%$. It is the third highest cause of mortality after acquired immune deficiency syndrome and tuberculosis [2]. Although ocular involvement in melioidosis is rare, the effects on patients' vision are devastating. Most patients with this condition ended up becoming legally blind. In our series of 16 cases, there were only 5 (36\%) in which patients had improved vision after treatment.

We suspect that the number of ocular melioidosis cases might be underestimated. Most of the melioidosis patients admitted to the hospital had disseminated septicemic melioidosis and were treated for life-threatening symptoms. Mild ocular symptoms might be easily overlooked, and ophthalmologists were not consulted in all cases.

The prevalence of ocular melioidosis in Srinagarind Hospital (1.02\%) was about twice that in Khon Kaen Hospital (0.49\%). The discrepancy might be due to the differences between the two hospitals. Srinagarind Hospital is the largest university hospital in northeast Thailand, and many severe cases of systemic melioidosis are referred to Srinagarind Hospital. Since more organs are affected in severe disseminated melioidosis, ocular involvement is more likely in these cases.

We suspect that the recent prevalence of ocular melioidosis in Srinagarind Hospital might be much higher than what we have found. From 2007 to April 2016, there were 264 cases of melioidosis at Srinagarind Hospital, of which 13 had ocular involvement. According to this finding, the prevalence during this time interval was as high as $4.9 \%$ (95\% confidence interval from 2.82 to $8.32 \%$ ).

This study led to some interesting findings. Ten patients (63\%) presented with eye symptoms, which later resulted in

Table 1 Data collection and prevalence $(95 \% \mathrm{Cl})$ calculation

\begin{tabular}{lllll}
\hline Tertiary hospital & Srinagarind University Hospital & Khon Kaen Provincial Hospital & Total from all data & Total from 2012 to 2016 \\
\hline Date & January 1993 to April 2016 & January 2012 to April 2016 & January 1993 to April 2016 January 2012 to April 2016 \\
Length & 23 years and 4 months & 4 years and 4 months & 23 years and 4 months & 4 years and 4 months \\
$\begin{array}{l}\text { Total melioidosis (cases) } \\
\text { Total ocular involvement (cases) }\end{array}$ & 1270 & 607 & 1877 & 859 \\
$\begin{array}{l}\text { Prevalence } \\
(95 \% \mathrm{Cl})\end{array}$ & $1.02 \%(0.58,1.76 \%)$ & $0.49 \%(0.10,0.51 \%)$ & $0.85 \%(0.51,1.39 \%)$ & 8 \\
\hline
\end{tabular}




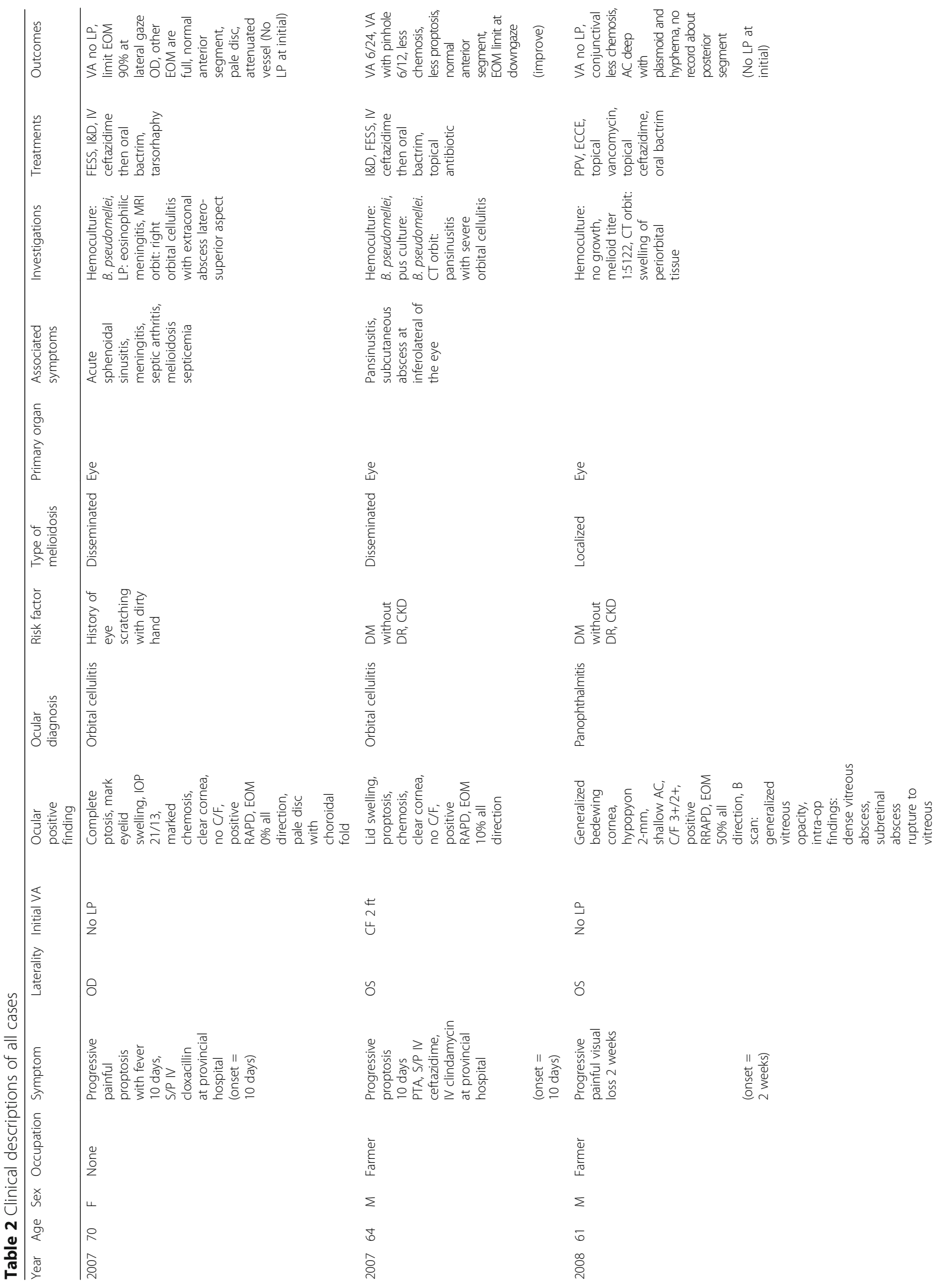




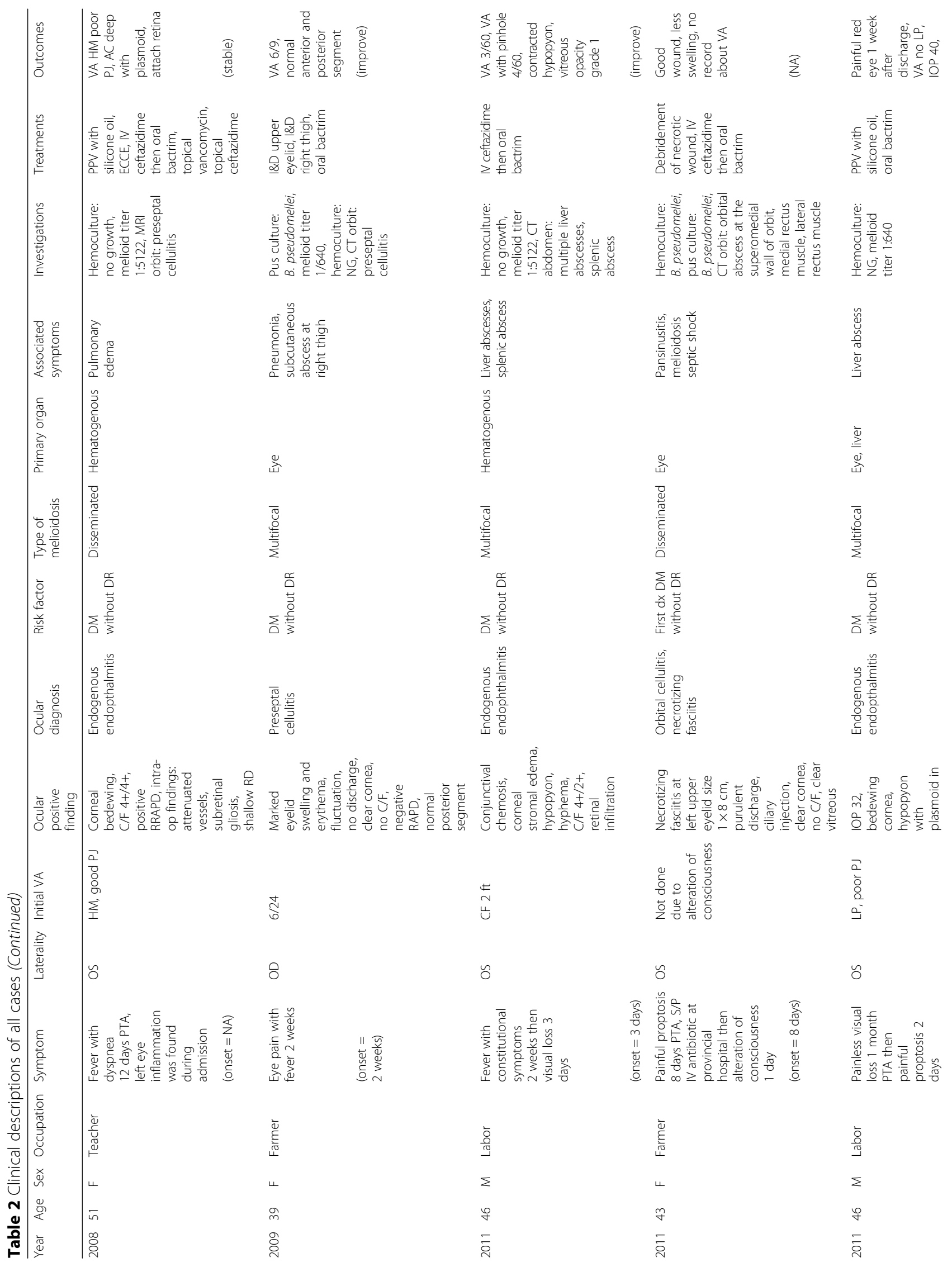




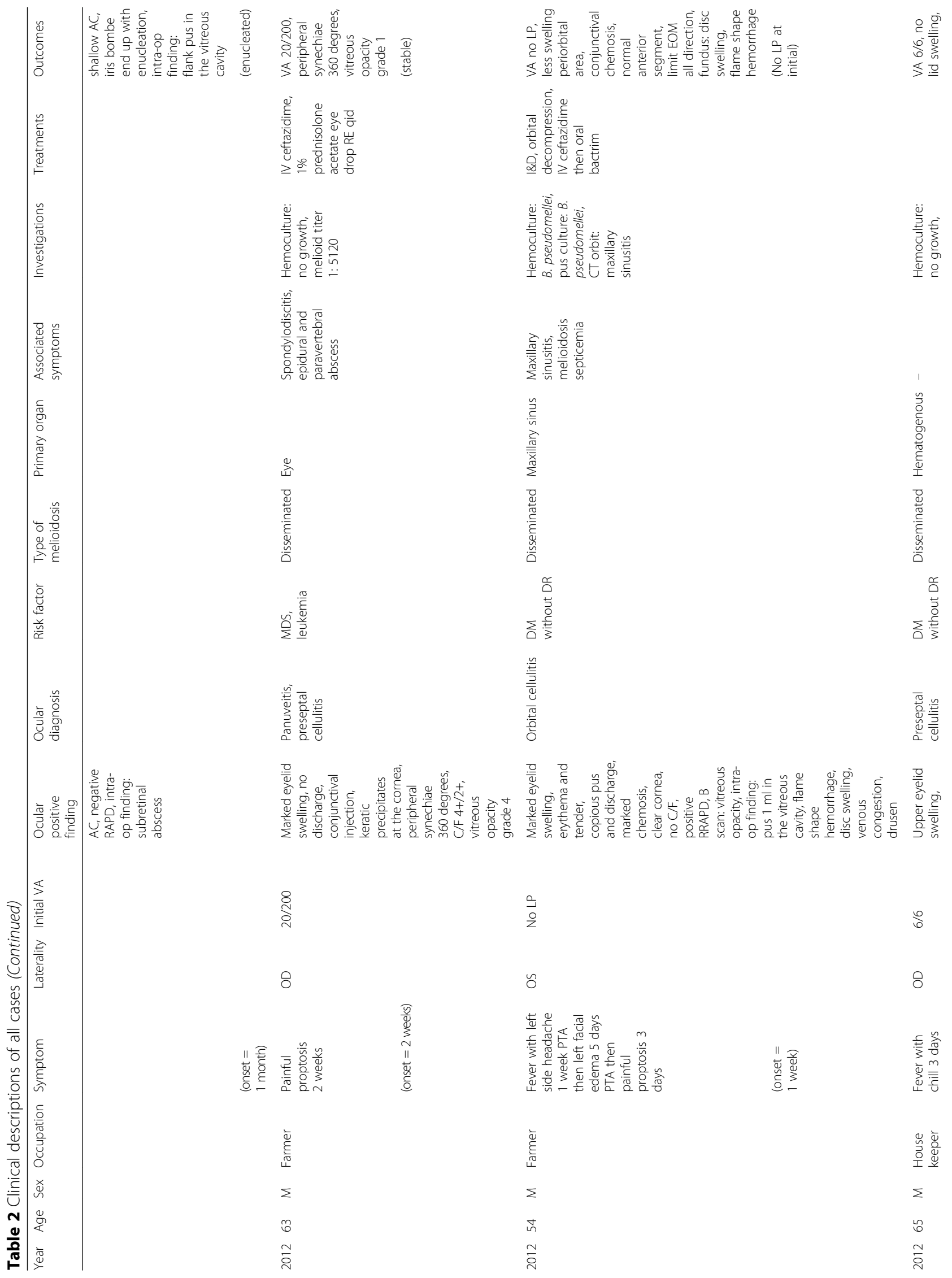




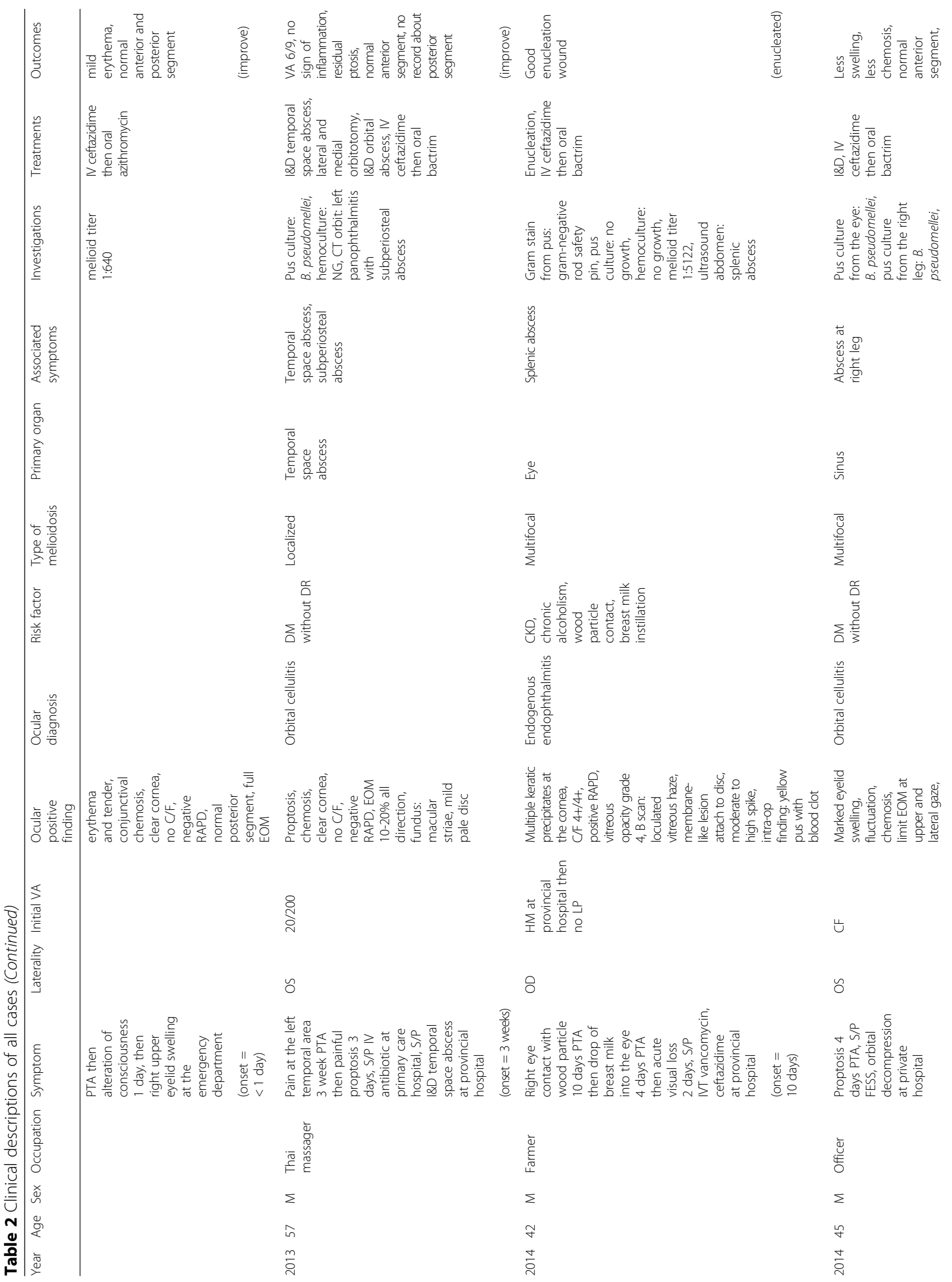




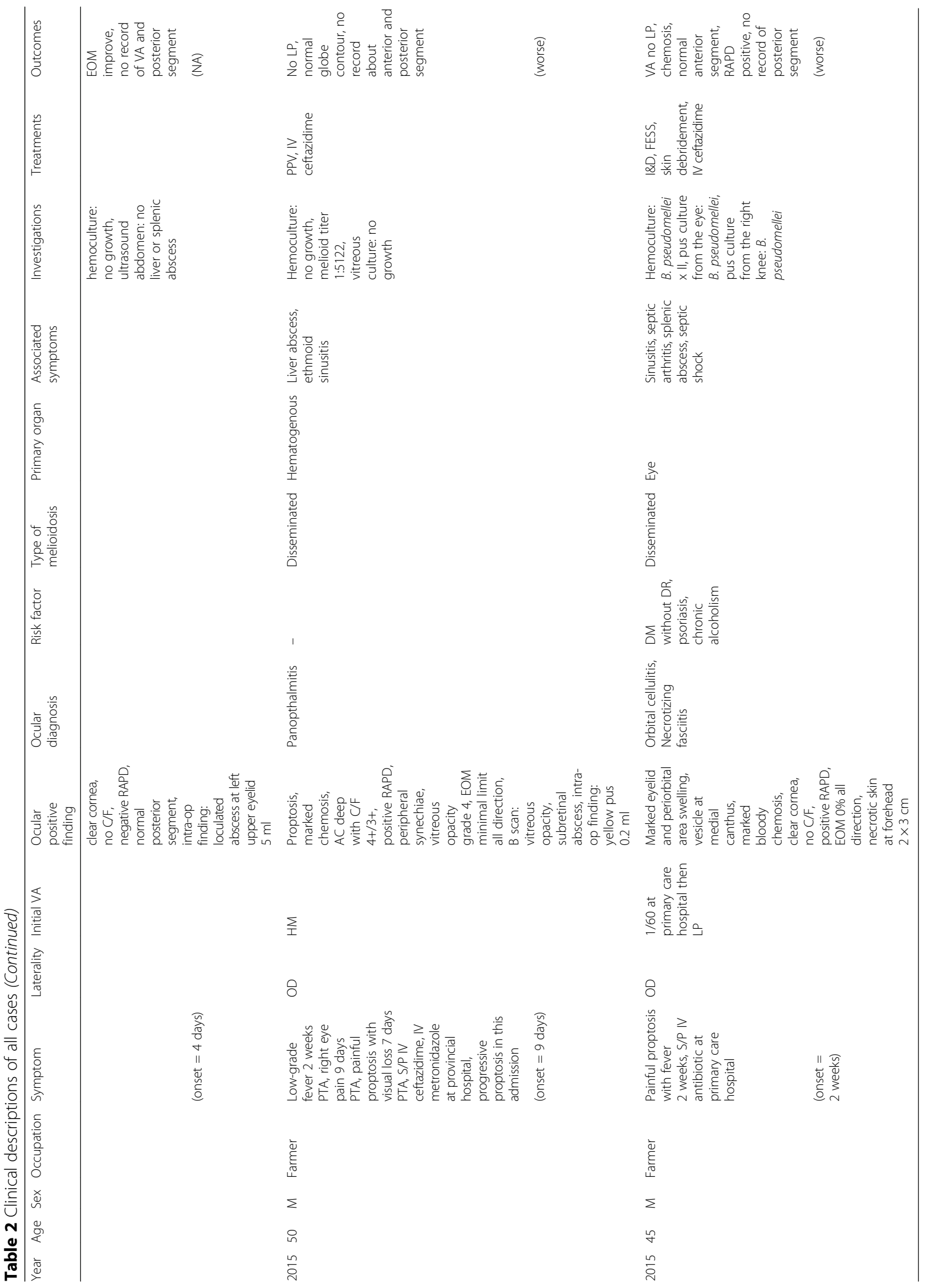


systemic spreading. On the other hand, there were six patients $(38 \%)$ whose first symptoms were not eye symptoms; four patients (25\%) presented with fever and two (13\%) presented with a headache. In most cases, diabetes mellitus was the underlying disease (12 cases, $75 \%$ ), but none of the patients in those cases had diabetic retinopathy.

Interestingly, we found that most cases of ocular melioidosis were classified as disseminated septicemic melioidosis (nine cases, 56\%) which means that there was a bloodstream infection. This is unlike other gram-positive organisms, which usually cause orbital cellulitis and commonly result in a negative hemoculture. The explanation for this finding may be attributable to the nature of Bulkholderia pseudomallei infection, which generally presents with bloodstream infection.

In our study, orbital cellulitis was the most common manifestation (seven cases, 44\%). Usually, orbital cellulitis is caused by gram-positive organisms and can be cured only by intravenous antibiotics, unlike orbital cellulitis caused by melioidosis. All of these patients ended up undergoing surgical intervention (100\%). The abscess-forming activity of Burkholderia pseudomallei may be the reason why intravenous antibiotics alone did not work to treat the condition.

Moreover, there were two cases (29\%) of orbital cellulitis that progressed to necrotizing fasciitis, which is uncommon in other types of bacterial orbital cellulitis. This is similar to the results of a previous case report by Saonanon P [13]. Unfortunately, all of our patients (100\%) with necrotizing fasciitis subsequently developed septic shock. Early suspicion and prompt surgical debridement may improve mortality in these patients.

We also found that even if systemic ceftazidime was used, the occurrence of endogenous endophthalmitis caused by melioidosis was not preventable, as stated in a previous report [10]. Most of the cases diagnosed as endophthalmitis and panophthalmitis required surgical intervention (five out of six cases, 83\%), including pars plana vitrectomy (three out of five cases, 60\%) and enucleation (two out of five cases, $40 \%$ ).

Two cases $(50 \%)$ of endophthalmitis were enucleated. The first case, from 2011, had a delayed presentation. The patient had experienced loss of vision for 1 month prior to admission, which was the longest onset in any of the cases. In the second case, from 2014, the patient exhibited two risk factors for the condition, including wood particle contact and breast milk instillation into the eye, as a result of local traditional treatment practices.

There were three cases that were cured without any surgical intervention. In one case, this was due to the patient seeking early treatment for endogenous endophthalmitis. The other two patients had diagnoses that did not require an operation (namely, panuveitis and preseptal cellulitis).

\section{Conclusions}

In summary, ocular involvement in melioidosis was rare, but the outcomes were devastating. The most common ocular involvements were orbital cellulitis and endophthalmitis. The morbidity in these cases was high, so it is critical to employ a high index of suspicion. Ocular melioidosis should be considered when the ocular infection does not respond to conventional antibiotic therapy, especially in hyperendemic regions for melioidosis. Early consultation with an ophthalmologist and prompt surgical intervention may significantly improve the final visual outcomes, as well as mortality rates.

\section{Methods}

We retrospectively reviewed all admitted cases of melioidosis with ocular involvement from two tertiary hospitals in Khon Kaen using electronic databases. The first is Srinagarind Hospital, which is a university hospital. We searched the hospital's electronic database for cases of this condition from January 1993 to April 2016 (23 years and 4 months). The second is Khon Kaen Hospital, which is a provincial hospital. We searched the hospital's electronic database for cases that presented between January 2012 and April 2016 (4 years and 4 months). The data were retrieved using the ICD10 code for melioidosis (all A24 codes) and all diseases of the eye and adnexa (code H00 to H59).

This manuscript adheres to the guidelines and principles laid out in the Declaration of Helsinki. Institutional review board (IRB) approval was obtained from the Khon Kaen University and Khon Kaen Hospital, Thailand. The clinical trial was registered in Thai Clinical Trials Registry (study ID: TCTR20160818004).

We only included cases in which there were positive cultures for melioidosis or high blood titer according to indirect hemagglutination (IHA). The cutoff point for positive antibody titers has been determined to be 1:160 in endemic areas [15]. Irrelevant ocular diagnoses, such as cataracts, glaucoma, diabetic retinopathy, or other underlying eye diseases, were excluded. The prevalence and 95\% confidence intervals $(95 \% \mathrm{CI})$ were calculated using the modified Wald method. Other results were summarized as proportions and percentages.

\footnotetext{
Abbreviations

AC: Anterior chamber; B. pseudomellei: Bulkholderia pseudomallei; C/F: Cell/ flare; CF: Counting fingers; CKD: Chronic kidney disease; DM: Diabetes mellitus; DR: Diabetic retinopathy; ECCE: Extracapsular cataract extraction; EOM: Extraocular movement; F: Female; FESS: Functional endoscopic sinus surgery; HM: Hand motion; I\&D: Incision and drainage; IOP: Intraocular pressure; LP: Light perception; LP: Lumbar puncture; M: Male; MDS: Myelodysplastic syndrome; MRI: Magnetic resonance imaging; NA: Not available; OD: Right eye; OS: Left eye; PJ: Light projection; PPV: Pars plana vitrectomy; RAPD: Relative afferent pupillary defect; RD: Retinal detachment: RRAPD: Reverse relative afferent pupillary defect; VA: Visual acuity
} 


\section{Acknowledgements}

We would like to thank our clinical colleagues at the Srinagarind hospital and Khon Kaen Hospital for their expertise with regard to the detection of ocular involvement, diagnosis, and management of the patients in this study. We would also like to thank our coders for the complete diagnosis records that lead to the discoveries described in this paper.

\section{Funding}

This work was supported by the Faculty of Medicine, Khon Kaen University.

\section{Authors' contributions}

SY carried out the ophthalmology studies, participated in the research design, participated in the data acquisition at the university hospital, participated in the data interpretation, and drafted the manuscript. SA carried out the ophthalmology practices, participated in the research design, participated in the statistical analysis, and helped to draft the manuscript. PC carried out the infectious practices, provided expertise regarding melioidosis, participated in the research design, helped facilitate the coordination between two hospitals in the study, and drafted the manuscript. SW carried out the microbiological studies, provided expertise with regard to melioidosis, participated in the data acquisition and coordination between departments, and helped to draft the manuscript. PP carried out the ophthalmology practices at the provincial hospital and participated in the data acquisition at the provincial hospital. All authors read and approved the final manuscript.

\section{Ethics approval and consent to participate}

The manuscript adheres to the guidelines and principles by the Declaration of Helsinki. Institutional review board (IRB) approval was obtained from Khon Kaen University, Thailand, numbered HE581497 and Khon Kaen Hospital, Thailand, numbered KE59045.

The clinical trial was registered in Thai Clinical Trials Registry study ID: TCTR20160818004.

\section{Consent for publication}

Not applicable

\section{Competing interests}

The authors declare that they have no competing interests.

\section{Publisher's Note}

Springer Nature remains neutral with regard to jurisdictional claims in published maps and institutional affiliations.

\section{Author details}

'Department of Ophthalmology, Faculty of Medicine, Khon Kaen University, Khon Kaen, Thailand. ${ }^{2}$ Department of Medicine, Faculty of Medicine, Khon Kaen University, Khon Kaen, Thailand. ${ }^{3}$ Melioidosis Research Center, Khon Kaen University, Khon Kaen, Thailand. ${ }^{4}$ Department of Microbiology, Faculty of Medicine, Khon Kaen University, Khon Kaen, Thailand. ${ }^{5}$ Department of Ophthalmology, Khon Kaen Hospital, Khon Kaen, Thailand.

Received: 5 October 2017 Accepted: 6 March 2018

Published online: 27 March 2018

\section{References}

1. Wiersinga WJ, Currie BJ, Peacock SJ (2012) Melioidosis. N Engl J Med 367: 1035-1044

2. Limmathurotsakul D, Wongratanacheewin S, Teerawattanasook $\mathrm{N}$ et al (2010) Increasing incidence of human melioidosis in northeast Thailand. Am J Trop Med Hyg 82:1113-1117

3. Yospaiboon Y, Sangveejit J (1987) Orbital cellulitis due to Pseudomonas pseudomallei. Thai J Ophthalmol 1:51-54

4. Suwanwatana C (1990) Acute dacrocystitis due to Pseudomonas pseudomallei. Srinakarin Hosp Med J 5:56-58

5. Siripanthong S, Teerapantuwat S, Prugsanusak W et al (1991) Corneal ulcer caused by Pseudomonas pseudomallei: report of three cases. Rev Infect Dis 13:335-337

6. Wong PK, Ng PH (1996) Melioidosis presenting with orbital cellulitis. Singap Med J 37:220-221
7. Srimuang S et al (1996) Immunobiological diagnosis of tropical ocular diseases: Toxocara, Pythium insidiosum, Pseudomonas (Burkholderia) pseudomallei, Mycobacterium chelonei and Toxoplasma gondii. Int J Tissue React 18(1):23-25

8. Abdul Rani MF, Samad Cheung H, Mohd. Shah A, Mahmood T (2002) Melioidosis presenting as orbital and parotid abscesses with intracranial extension. Int Med J Malaysia 1(2).www.e-imj.com

9. Yang $\mathbb{H}$, Lee JJ, Liu JW et al (2006) Melioidosis with endophthalmitis. Arch Ophthalmol 124:1501-1502

10. Chen K, Sun M, Hou C, Sun C, Chen T (2007) Burkholderia pseudomallei endophthalmitis. J Clin Microbiol 45(12):4073-4074

11. Shawarinin J, Bakiah S, Shatriah I (2009) Successfully treated rare presentation of orbital melioidosis. Int J Ophthalmol 2:90-92

12. Tirakunwichcha S, Vaivanijkul J (2009) Melioidosis presenting as orbital apex syndrome. Asian J Ophthalmol 11:40-42

13. Saonanon P, Tirakunwichcha S, Chierakul W (2013) Case report of orbital cellulitis and necrotizing fasciitis from melioidosis. Ophthal Plast Reconstr Surg 29(3):e81-e84

14. Kogilavaani J, Shatriah I, Regunath K, Helmy A (2014) Bilateral orbital abscesses with subdural empyema and cavernous sinus thrombosis due to melioidosis in a child. Asian Pac J Trop Dis 4:S851-\$853

15. Naigowit P, Maneeboonyoung W, Wongroonsub P et al (1992) Serosurveillance for Pseudomonas pseudomallei infection in Thailand. Jpn J Med Sci Biol 45:215-230

\section{Submit your manuscript to a SpringerOpen ${ }^{\circ}$ journal and benefit from:}

- Convenient online submission

- Rigorous peer review

- Open access: articles freely available online

- High visibility within the field

- Retaining the copyright to your article

Submit your next manuscript at springeropen.com 